In: Integral Methods in Science and Engineering (edited by C. Constanda, M. Pérez), volume 1: Analytic Methods, Birkhäuser, Boston-Basel-Berlin (2010), ISBN 978-08176-4898-5, 29-39.

\title{
Two-Operator Boundary-Domain Integral Equations for a Variable-Coefficient BVP
}

\author{
T.G. Ayele ${ }^{1}$ and S.E. Mikhailov ${ }^{2}$ \\ ${ }^{1}$ Addis Ababa University, Ethiopia; tsegayeg@math.aau.edu.et \\ ${ }^{2}$ Brunel University West London, UK; sergey.mikhailov@brunel.ac.uk
}

\subsection{Introduction}

Partial Differential Equations (PDEs) with variable coefficients often arise in mathematical modelling of inhomogeneous media (e.g. functionally graded materials or materials with damage induced inhomogeneity) in solid mechanics, electromagnetics, thermo-conductivity, fluid flows trough porous media, and other areas of physics and engineering.

Generally, explicit fundamental solutions are not available if the PDE coefficients are not constant, preventing formulation of explicit boundary integral equations for them, which can then be effectively solved numerically. Nevertheless, for a rather wide class of variable-coefficient PDEs it is possible to use instead an explicit parametrix (Levi function) taken as a fundamental solution of corresponding frozen-coefficient PDEs, and reduce Boundary Value Problems (BVPs) for such PDEs to explicit systems of Boundary-Domain Integral Equations (BDIEs), see e.g. [Mi02, CMN09, Mi06] and references therein. However this (one-operator) approach does not work when the fundamental solution of the frozen-coefficient PDE is not available explicitly (as e.g. in the Lamé system of anisotropic elasticity).

To overcome this difficulty, one can apply the so-called two-operator approach, formulated in [Mi05] for some non-linear problems, that employs a parametrix of another (second) PDE, not related with the PDE in question, for reducing the BVP to a BDIE system. Since the second PDE is rather arbitrary, one can always chose it by such a way, that its parametrix is available explicitly. A simplest choice for the second PDE is the one with a fundamental solution explicitly available.

To analyse the two-operator approach we apply in this paper one of its linear versions to the mixed (Dirichlet-Neumann) BVP for a linear second-order scalar elliptic variable-coefficient PDE reducing it to four different BDIE systems. Although the considered BVP can be reduced to (other) BDIE systems also by the one-operator approach, it can be considered as a simple "toy" 
model showing the main features of the two-operator approach arising also in reducing more general BVPs to BDIEs. The two-operator BDIE systems are nonstandard systems of equations containing integral operators defined on the domain under consideration and potential type and pseudo-differential operators defined on open sub-manifolds of the boundary. Using the results of [CMN09], we give a rigorous analysis of the two-operator BDIEs and show that the BDIE systems are equivalent to the mixed BVP and thus are uniquely solvable, while the corresponding boundary domain integral operators are invertible in appropriate Sobolev-Slobodetski (Bessel-potential) spaces.

\subsection{Function spaces and BVP}

Let $\Omega=\Omega^{+}$be an open three-dimensional region of $\mathbb{R}^{3}, \Omega^{-}:=\mathbb{R}^{3} \backslash \overline{\Omega^{+}}$and the boundary $\partial \Omega$ be simply connected, closed, infinitely smooth surface. Moreover, $\partial \Omega=\overline{\partial_{D} \Omega} \bigcup \overline{\partial_{N} \Omega}$ where $\partial_{D} \Omega$ and $\partial_{N} \Omega$ are open, non-empty, nonintersecting, simply connected sub-manifolds of $\partial \Omega$ with an infinitely smooth boundary curve $\overline{\partial_{D} \Omega} \cap \overline{\partial_{N} \Omega} \in C^{\infty}$. Let $a \in C^{\infty}\left(\mathbb{R}^{3}\right), a(x)>0$ and also $\partial_{j}:=\partial / \partial x_{j}(j=1,2,3), \partial_{x}=\left(\partial_{1}, \partial_{2}, \partial_{3}\right)$. We consider the following PDE with scalar variable coefficient,

$$
L_{a} u(x):=L_{a}\left(x, \partial_{x}\right) u(x):=\sum_{i=1}^{3} \frac{\partial}{\partial x_{i}}\left[a(x) \frac{\partial u(x)}{\partial x_{i}}\right]=f(x), \quad x \in \Omega^{ \pm},
$$

where $u$ is unknown function and $f$ is a given function in $\Omega^{ \pm}$.

In what follows, $H^{s}\left(\Omega^{+}\right)=H_{2}^{s}\left(\Omega^{+}\right), H_{l o c}^{s}\left(\Omega^{-}\right)=H_{2, l o c}^{s}\left(\Omega^{-}\right), H^{s}(\partial \Omega)=$ $H_{2}^{s}(\partial \Omega)$ denote the Bessel potential spaces (coinciding with the SobolevSlobodetski spaces if $s \geq 0$ ). For $S_{1} \subset \partial \Omega$, we will use the subspace $\widetilde{H}^{s}\left(S_{1}\right)=\left\{g: g \in H^{s}(\partial \Omega), \operatorname{supp}(g) \subset \overline{S_{1}}\right\}$ of $H^{s}(\partial \Omega)$, while $H^{s}\left(S_{1}\right)=$ $\left\{r_{S_{1}} g: g \in H^{s}(\partial \Omega)\right\}$, where $r_{S_{1}}$ denotes the restriction operator on $S_{1}$.

From the trace theorem (see, e.g., [LiMa72]) for $u \in H^{1}\left(\Omega^{ \pm}\right)$, it follows that $\left.u\right|_{\partial \Omega} ^{ \pm}:=\gamma^{ \pm} u \in H^{\frac{1}{2}}(\partial \Omega)$, where $\gamma^{ \pm}$is the trace operator on $\partial \Omega$ from $\Omega^{ \pm}$. We will use $\gamma$ for $\gamma^{ \pm}$if $\gamma^{+}=\gamma^{-}$. We will use also notations $u^{ \pm}$for the traces $\left.u\right|_{\partial \Omega} ^{ \pm}$, when this will cause no confusion.

For a linear operator $L_{*}$ we introduce the following subspace of $H^{s}\left(\Omega^{ \pm}\right)$, [Gr85, Co88],

$$
\begin{gathered}
H^{s, 0}\left(\Omega^{ \pm} ; L_{*}\right):=\left\{g \in H^{s}\left(\Omega^{ \pm}\right): L_{*} g \in L_{2}\left(\Omega^{ \pm}\right)\right\}, \\
\|g\|_{H^{s, 0}\left(\Omega^{ \pm} ; L_{*}\right)}^{2}:=\|g\|_{H^{s}}^{2}+\left\|L_{*} g\right\|_{H^{0}\left(\Omega^{ \pm}\right)}^{2}=\|g\|_{H^{s}}^{2}+\left\|L_{*} g\right\|_{L_{2}\left(\Omega^{ \pm}\right)}^{2} .
\end{gathered}
$$

In this paper, we will particularly use the space $H^{1,0}\left(\Omega^{ \pm} ; L_{*}\right)$ for $L_{*}$ being either the operator $L_{a}$ from (4.1) or the Laplace operator $\Delta$, and one can see that these spaces coincide.

For $u \in H^{1,0}\left(\Omega^{ \pm} ; \Delta\right)$, we can correctly define the (canonical) co-normal derivative $T_{a}^{ \pm} u \in H^{-\frac{1}{2}}(\partial \Omega)$, cf. [Co88, McL00, Mi07], as 


$$
\left\langle T_{a}^{ \pm} u, w\right\rangle_{\partial \Omega}:= \pm \int_{\Omega^{ \pm}}\left[\gamma_{-1}^{ \pm} w \cdot L_{a} u+E_{a}\left(u, \gamma_{-1}^{ \pm} w\right)\right] d x \quad \forall w \in H^{1 / 2}(\partial \Omega),
$$

where $\gamma_{-1}^{ \pm}: H^{1 / 2}(\partial \Omega) \rightarrow H^{1}(\Omega \pm)$ is a right inverse to the trace operator $\gamma^{ \pm}$

$$
E_{a}(u, v):=\sum_{i=1}^{3} a(x) \frac{\partial u(x)}{\partial x_{i}} \frac{\partial v(x)}{\partial x_{i}}=a(x) \nabla u(x) \cdot \nabla v(x)
$$

and $\langle\cdot, \cdot\rangle_{\partial \Omega}$ denotes the duality brackets between the spaces $H^{-\frac{1}{2}}(\partial \Omega)$ and $H^{\frac{1}{2}}(\partial \Omega)$, which extend the usual $L_{2}(\partial \Omega)$ inner product; to simplify notations we will also write sometimes the duality brackets as integral. Then for $u \in$ $H^{1,0}\left(\Omega^{ \pm} ; \Delta\right), v \in H^{1}(\Omega)$ the first Green identity holds, [Co88, Lemma3.4], [Mi07, Lemma 4.8],

$$
\int_{\Omega^{ \pm}} v(x) L_{a} u(x) d x= \pm \int_{\partial \Omega} v(x) T_{a}^{+} u(x) d S(x)-\int_{\Omega^{ \pm}} E_{a}(u, v) d x .
$$

If $u \in H^{2}\left(\Omega^{ \pm}\right)$, the canonical co-normal derivative $T_{a}^{ \pm} u$ defined by (4.2) reduces to its classical form

$$
T_{a}^{ \pm} u:=\sum_{i=1}^{3} a(x) n_{i}(x)\left[\frac{\partial u(x)}{\partial x_{i}}\right]^{ \pm}=a(x)\left[\frac{\partial u(x)}{\partial n(x)}\right]^{ \pm},
$$

where $n(x)$ is the exterior (to $\Omega^{ \pm}$) unit normal at the point $x \in \partial \Omega$.

We will derive and investigate the two-operator boundary-domain integral equation systems for the following mixed boundary value problem.

$$
\begin{aligned}
L_{a} u & =f & & \text { in } \Omega \\
u^{+} & =\varphi_{0} & & \text { on } \partial_{D} \Omega \\
T_{a}^{+} u & =\psi_{0} & & \text { on } \partial_{N} \Omega
\end{aligned}
$$

where $\varphi_{0} \in H^{\frac{1}{2}}\left(\partial_{D} \Omega\right), \psi_{0} \in H^{-\frac{1}{2}}\left(\partial_{N} \Omega\right)$ and $f \in L_{2}(\Omega)$. Equation (4.5) is understood in the distributional sense, condition (4.6) in the trace sense, while equality (4.7) in the functional sense (4.2).

Let us consider another auxiliary linear elliptic partial differential operator $L_{b}$ such that

$$
L_{b} u(x):=L_{b}\left(x, \partial_{x}\right) u(x):=\sum_{i=1}^{3} \frac{\partial}{\partial x_{i}}\left[b(x) \frac{\partial u(x)}{\partial x_{i}}\right],
$$

where $b \in C^{\infty}\left(\mathbb{R}^{3}\right), b(x)>0$. Then for $u \in H^{1,0}\left(\Omega^{ \pm} ; \Delta\right)=H^{1,0}\left(\Omega^{ \pm} ; \Delta\right)$ the associate co-normal derivative operator $T_{b}^{ \pm}$is defined by (4.2) (and for $u \in H^{2}\left(\Omega^{ \pm}\right)$by (4.4)) with $a$ replaced by $b$. If $v \in H^{1,0}\left(\Omega^{ \pm} ; \Delta\right), u \in H^{1}(\Omega)$ then for the operator $L_{b}$ holds the first Green identity, 


$$
\int_{\Omega^{ \pm}} u(x) L_{b} v(x) d x= \pm \int_{\partial \Omega} u(x) T_{b}^{ \pm} v(x) d S-\int_{\Omega^{ \pm}} E_{b}(u, v) d x .
$$

If $u, v \in H^{1,0}\left(\Omega^{ \pm} ; \Delta\right)$, then subtracting (4.3) from (4.9), we obtain the twooperator second Green identity, cf. [Mi05],

$$
\begin{aligned}
& \int_{\Omega^{ \pm}}\left\{u(x) L_{b} v(x)\right.\left.-v(x) L_{a} u(x)\right\} d x= \\
& \pm \int_{\partial \Omega}\left\{u(x) T_{b}^{+} v(x)-v(x) T_{a}^{+} u(x)\right\} d S \\
& \quad+\int_{\Omega^{ \pm}}[a(x)-b(x)] \nabla v(x) \cdot \nabla u(x) d x
\end{aligned}
$$

Note that if $a=b$, then, the last domain integral disappears, and the twooperator Green identity degenerates into the classical second Green identity.

\subsection{Parametrix and potential type operators}

As follows from [Mir70, Mi02, CMN09], the function

$$
P_{b}(x, y)=\frac{-1}{4 \pi b(y)|x-y|}, \quad x, y \in \mathbb{R}^{3}
$$

is a parametrix (Levi function) for the operator $L_{b}\left(x ; \partial_{x}\right)$ from (4.8), i.e., satisfies equation

$$
L_{b}\left(x, \partial_{x}\right) P_{b}(x, y)=\delta(x-y)+R_{b}(x, y)
$$

with

$$
R_{b}(x, y)=\sum_{i=1}^{3} \frac{x_{i}-y_{i}}{4 \pi b(y)|x-y|^{3}} \frac{\partial b(x)}{\partial x_{i}}, \quad x, y \in \mathbb{R}^{3} .
$$

Evidently, the parametrix $P_{b}(x, y)$ is a fundamental solution to the operator $L_{b}\left(y, \partial_{x}\right):=b(y) \Delta\left(\partial_{x}\right)$ with "frozen" coefficient $b(x)=b(y)$, i.e.,

$$
L_{b}\left(y, \partial_{x}\right) P_{b}(x, y)=\delta(x-y) .
$$

The parametrix-based Newtonian and the remainder volume potential operators, corresponding to the parametrix (4.11) and to remainder (4.12) are given, respectively, by

$$
\mathcal{P}_{b} g(y):=\int_{\Omega} P_{b}(x, y) g(x) d x, \quad \mathcal{R}_{b} g(y):=\int_{\Omega} R_{b}(x, y) g(x) d x .
$$

Let us introduce the single layer and the double layer surface potential operators, based on parametrix (4.11), 


$$
\begin{array}{ll}
V_{b} g(y):=-\int_{\partial \Omega} P_{b}(x, y) g(x) d S_{x}, & y \notin \partial \Omega, \\
W_{b} g(y):=-\int_{\partial \Omega}\left[T_{b}\left(x, n(x), \partial_{x}\right) P_{b}(x, y)\right] g(x) d S_{x}, & y \notin \partial \Omega .
\end{array}
$$

For $y \in \partial \Omega$, the corresponding boundary integral (pseudo-differential) operators of direct surface values of the simple layer potential $\mathcal{V}_{b}$ and the double layer potential $\mathcal{W}_{b}$ are

$$
\begin{aligned}
\mathcal{V}_{b} g(y) & :=-\int_{\partial \Omega} P_{b}(x, y) g(x) d S_{x}, \\
\mathcal{W}_{b} g(y) & :=-\int_{\partial \Omega}\left[T_{b}\left(x, n(x), \partial_{x}\right) P_{b}(x, y)\right] g(x) d S_{x} .
\end{aligned}
$$

We can also calculate at $y \in \partial \Omega$ the co-normal derivatives, associated with the operator $L_{a}$, of the single layer potential and of the double layer potential,

$$
\begin{aligned}
T_{a}^{ \pm} V_{b} g(y) & =\frac{a(y)}{b(y)} T_{b}^{ \pm} V_{b} g(y), \\
\mathcal{L}_{a b}^{ \pm} g(y):=T_{a}^{ \pm} W_{b} g(y) & =\frac{a(y)}{b(y)} T_{b}^{ \pm} W_{b} g(y)=: \frac{a(y)}{b(y)} \mathcal{L}_{b}^{ \pm} g(y)
\end{aligned}
$$

The direct value operators associated with (4.18) are

$$
\begin{aligned}
\mathcal{W}_{a b}^{\prime} g(y) & :=-\int_{\partial \Omega}\left[T_{a}\left(y, n(y), \partial_{y}\right) P_{b}(x, y)\right] g(x) d S_{x}=\frac{a(y)}{b(y)} \mathcal{W}_{b}^{\prime} g(y), \\
\mathcal{W}_{b}^{\prime} g(y) & :=-\int_{\partial \Omega}\left[T_{b}\left(y, n(y), \partial_{y}\right) P_{b}(x, y)\right] g(x) d S_{x}
\end{aligned}
$$

From equations (4.13)-(4.21) we deduce representations of the parametrixbased surface potential boundary operators in terms of their counterparts for $b=1$, that is, associated with the fundamental solution $P_{\Delta}=-(4 \pi|x-y|)^{-1}$ of the Laplace operator $\Delta$.

$$
\begin{gathered}
\mathcal{P}_{b} g=\frac{1}{b} \mathcal{P}_{\Delta} g, \quad \mathcal{R}_{b} g=-\frac{1}{b} \sum_{j=1}^{3} \partial_{j} \mathcal{P}_{\Delta}\left[g\left(\partial_{j} b\right)\right], \\
\frac{a}{b} V_{a} g=V_{b} g=\frac{1}{b} V_{\Delta} g ; \quad \frac{a}{b} W_{a}\left(\frac{b g}{a}\right)=W_{b} g=\frac{1}{b} W_{\Delta}(b g), \\
\frac{a}{b} \mathcal{V}_{a} g=\mathcal{V}_{b} g=\frac{1}{b} \mathcal{V}_{\Delta} g ; \quad \frac{a}{b} \mathcal{W}_{a}\left(\frac{b g}{a}\right)=\mathcal{W}_{b} g=\frac{1}{b} \mathcal{W}_{\Delta}(b g), \\
\mathcal{W}^{\prime}{ }_{a b} g=\frac{a}{b} \mathcal{W}_{b}^{\prime} g=\frac{a}{b}\left\{\mathcal{W}_{\Delta}^{\prime}(b g)+\left[b \frac{\partial}{\partial n}\left(\frac{1}{b}\right)\right] \mathcal{V}_{\Delta} g\right\}, \\
\mathcal{L}_{a b}^{ \pm} g:=\frac{a}{b} \mathcal{L}_{b}^{ \pm} g=\frac{a}{b}\left\{\mathcal{L}_{\Delta}(b g)+\left[b \frac{\partial}{\partial n}\left(\frac{1}{b}\right)\right] W_{\Delta}^{ \pm}(b g)\right\} .
\end{gathered}
$$


It is taken into account that $b$ and its derivatives are continuous in $\mathbb{R}^{3}$ and $\mathcal{L}_{\Delta}(b g):=\mathcal{L}_{\Delta}^{+}(b g)=\mathcal{L}_{\Delta}^{-}(b g)$ by the Liapunov-Tauber theorem.

The mapping properties of the volume and surface potentials are proved in [CMN09], see also Appendix A and B in [Mi06]. Similar to Theorems 3.3 and 3.6 in [CMN09] (see also Appendix A and B in [Mi06]), relations (4.23)-(4.26) imply the two following jump relation theorems.

Theorem 1. Let $g_{1} \in H^{-\frac{1}{2}}(\partial \Omega)$, and $g_{2} \in H^{\frac{1}{2}}(\partial \Omega)$. Then there hold the following relations on $\partial \Omega$,

$$
\begin{aligned}
{\left[V_{b} g_{1}\right]^{ \pm} } & =\mathcal{V}_{b} g_{1}, \\
{\left[W_{b} g_{2}\right]^{ \pm} } & =\mp \frac{1}{2} g_{2}+\mathcal{W}_{b} g_{2}, \\
T_{a}^{ \pm} V_{b} g_{1} & = \pm \frac{1}{2} \frac{a}{b} g_{1}+\mathcal{W}^{\prime}{ }_{a b} g_{1} .
\end{aligned}
$$

Theorem 2. Let $S_{1}$ and $\partial \Omega \backslash \bar{S}_{1}$ be nonempty, open, simply connected submanifolds of $\partial \Omega$ with an infinitely smooth boundary curve, and $0<s<1$. Then

$$
\mathcal{L}_{a b}^{+}+\frac{a}{b} \frac{\partial b}{\partial n}\left(-\frac{1}{2} I+\mathcal{W}_{b}\right)=\mathcal{L}_{a b}^{-}+\frac{a}{b} \frac{\partial b}{\partial n}\left(\frac{1}{2} I+\mathcal{W}_{b}\right) \text { on } \partial \Omega
$$

Moreover, the pseudo-differential operator $r_{S_{1}} \widehat{\mathcal{L}}_{a b}: \widetilde{H}^{s}\left(S_{1}\right) \rightarrow H^{s-1}\left(S_{1}\right)$, where

$$
\widehat{\mathcal{L}}_{a b} g:=\left[\frac{b}{a} \mathcal{L}_{a b}^{ \pm}+\frac{\partial b}{\partial n}\left(\mp \frac{1}{2} I+\mathcal{W}_{b}\right)\right] g=\mathcal{L}_{\Delta}(b g) \text { on } \partial \Omega,
$$

is invertible, while the operators $r_{S_{1}}\left(\frac{b}{a} \mathcal{L}_{a b}^{ \pm}-\widehat{\mathcal{L}}_{a b}\right): \widetilde{H}^{s}\left(S_{1}\right) \rightarrow H^{s}\left(S_{1}\right)$ are bounded and the operators $r_{S_{1}}\left(\frac{b}{a} \mathcal{L}_{a b}^{ \pm}-\widehat{\mathcal{L}}_{a b}\right): \widetilde{H}^{s}\left(S_{1}\right) \rightarrow H^{s-1}\left(S_{1}\right)$ are compact.

For $v(x):=P_{b}(x, y)$ and $u \in H^{1,0}(\Omega ; \Delta)$, we obtain from (4.10) by standard limiting procedures (cf. [Mir70]) the two-operator third Green identity,

$$
u+\mathcal{Z}_{b} u+\mathcal{R}_{b} u-V_{b} T_{a}^{+} u+W_{b} u^{+}=\mathcal{P}_{b} L_{a} u \quad \text { in } \Omega,
$$

where

$$
\begin{aligned}
\mathcal{Z}_{b} u(y):=-\int_{\Omega}[a(x)- & b(x)] \nabla_{x} P_{b}(x, y) \cdot \nabla u(x) d x \\
& =\frac{1}{b(y)} \sum_{j=1}^{3} \partial_{j} \mathcal{P}_{\Delta}\left[(a-b) \partial_{j} u\right](y), \quad y \in \Omega .
\end{aligned}
$$


Using the Gauss divergence theorem, we can rewrite $\mathcal{Z}_{b} u(y)$ in the form that does not involve derivatives of $u$,

$$
\begin{aligned}
& \mathcal{Z}_{b} u(y)=\left[\frac{a(y)}{b(y)}-1\right] u(y)+\widehat{\mathcal{Z}}_{b} u(y), \\
& \widehat{\mathcal{Z}}_{b} u(y):=\frac{a(y)}{b(y)} W_{a} u^{+}(y)-W_{b} u^{+}(y)+\frac{a(y)}{b(y)} \mathcal{R}_{a} u(y)-\mathcal{R}_{b} u(y),
\end{aligned}
$$

which allows to call $\mathcal{Z}_{b}$ integral operator in spite of its integro-differential ansatz (4.28).

Note that substituting (4.29)-(4.30) to (4.27) and multiplying by $b(y) / a(y)$ one reduces (4.27) to the one-operator parametrix-based third Green identity obtained in [CMN09],

$$
u+\mathcal{R}_{a} u-V_{a} T_{a}^{+} u+W_{a} u^{+}=\mathcal{P}_{a} L_{a} u \quad \text { in } \Omega .
$$

Relations (4.28)-(4.30) and the mapping properties of $\mathcal{P}_{\Delta}, \mathcal{R}_{a}, \mathcal{R}_{b}, W_{a}$ and $W_{b}$, given by Theorems 3.1, 3.8 in [CMN09], imply the following statement.

Theorem 3. The operators

$$
\begin{aligned}
& \mathcal{Z}_{b}: H^{s}(\Omega) \rightarrow H^{s}(\Omega), \quad s>\frac{1}{2}, \\
& \widehat{\mathcal{Z}}_{b}: H^{s}(\Omega) \rightarrow H^{s, 0}(\Omega ; \Delta), \quad s \geq 1,
\end{aligned}
$$

are continuous.

If $u \in H^{1,0}(\Omega ; \Delta)$ is a solution of equation (4.5) with $f \in L_{2}(\Omega)$, then (4.27) gives

$$
\begin{array}{r}
G u:=u+\mathcal{Z}_{b} u+\mathcal{R}_{b} u-V_{b} T_{a}^{+} u+W_{b} u^{+}=\mathcal{P}_{b} f \text { in } \Omega, \\
\mathcal{G} u:=\frac{1}{2} u^{+}+\mathcal{Z}_{b}^{+} u+\mathcal{R}_{b}^{+} u-\mathcal{V}_{b} T_{a}^{+} u+\mathcal{W}_{b} u^{+}=\left[\mathcal{P}_{b} f\right]^{+} \text {on } \partial \Omega, \\
\mathcal{T} u:=\left(1-\frac{a}{2 b}\right) T_{a}^{+} u+T_{a}^{+} \mathcal{Z}_{b} u+T_{a}^{+} \mathcal{R}_{b} u-\mathcal{W}^{\prime}{ }_{a b} T_{a}^{+} u \\
+\mathcal{L}_{a b}^{+} u^{+}=T_{a}^{+} \mathcal{P}_{b} f \text { on } \partial \Omega .
\end{array}
$$

where $\mathcal{Z}_{b}^{+} u=\left[\mathcal{Z}_{b} u\right]^{+}$and $\mathcal{R}_{b}^{+} u=\left[\mathcal{R}_{b} u\right]^{+}$.

Note that if $\mathcal{P}_{b}$ is not only the parametrix but also a fundamental solution of the operator $L_{b}$, then the remainder operator $\mathcal{R}_{b}$ vanishes in (4.31)-(4.33) (and everywhere in the paper), while the operator $\mathcal{Z}_{b}$ stays unless $L_{a}=L_{b}$.

For some functions $f, \Psi, \Phi$, let us consider a more general "indirect" integral relation, associated with (4.31),

$$
u+\mathcal{Z}_{b} u+\mathcal{R}_{b} u-V_{b} \Psi+W_{b} \Phi=\mathcal{P}_{b} f, \quad \text { in } \Omega
$$

Similar to the proof of Lemma 4.1 in [CMN09], one can prove the following

Lemma 1. Let $f \in L_{2}(\Omega), \Psi \in H^{-\frac{1}{2}}(\partial \Omega), \Phi \in H^{\frac{1}{2}}(\partial \Omega)$, and $u \in H^{1}(\Omega)$ satisfy (4.34). Then $u \in H^{1,0}(\Omega ; \Delta), L_{a} u=f$ in $\Omega$ and

$$
V_{b}\left(\Psi-T_{a}^{+} u\right)-W_{b}\left(\Phi-u^{+}\right)=0 \quad \text { in } \Omega .
$$




\subsection{Two-operator boundary-domain integral equations}

Let $\Phi_{0} \in H^{\frac{1}{2}}(\partial \Omega)$ and $\Psi_{0} \in H^{-\frac{1}{2}}(\partial \Omega)$ be some extensions of the given data $\varphi_{0} \in H^{\frac{1}{2}}\left(\partial_{D} \Omega\right)$ from $\partial_{D} \Omega$ to $\partial \Omega$ and $\psi_{0} \in H^{-\frac{1}{2}}\left(\partial_{N} \Omega\right)$ from $\partial_{N} \Omega$ to $\partial \Omega$, respectively. Let us also denote

$$
F_{0}:=\mathcal{P}_{b} f+V_{b} \Psi_{0}-W_{b} \Phi_{0} \quad \text { in } \quad \Omega .
$$

Note that for $f \in L_{2}(\Omega), \Psi_{0} \in H^{-\frac{1}{2}}(\partial \Omega)$ and $\Phi_{0} \in H^{\frac{1}{2}}(\partial \Omega)$, we have the inclusion $F_{0} \in H^{1,0}\left(\Omega, L_{a}\right)$ due to the mapping properties of the Newtonian (volume) and layer potentials (cf. Theorems 3.1 and 3.10 in [CMN09]).

To reduce BVP (4.5)-(4.7) to one or another two-operator BDIE system, we will use equation (4.31) in $\Omega$, and restrictions of equation (4.32) or (4.33) on appropriate parts of the boundary. We will always substitute $\Phi_{0}+\varphi$ for $u^{+}$ and $\Psi_{0}+\psi$ for $T_{a}^{+} u$, cf. [CMN09], where $\Phi_{0} \in H^{\frac{1}{2}}(\partial \Omega)$ and $\Psi_{0} \in H^{-\frac{1}{2}}(\partial \Omega)$ are considered as known, while $\psi$ belongs to $\widetilde{H}^{-\frac{1}{2}}\left(\partial_{D} \Omega\right)$ and $\varphi$ to $\widetilde{H}^{\frac{1}{2}}\left(\partial_{N} \Omega\right)$ due to the boundary conditions (4.6)-(4.7) and are to be found along with $u \in H^{1,0}(\Omega ; \Delta)$. This will lead us to segregated BDIE systems.

\subsubsection{Boundary-domain integral equation system $(\mathcal{G} \mathcal{T})$}

Let us use equation (4.31) in $\Omega$, the restriction of equation (4.32) on $\partial_{D} \Omega$ and the restriction of equation (4.33) on $\partial_{N} \Omega$. Then we arrive at the following twooperator segregated system of BDIEs,

$$
\begin{aligned}
& u+\mathcal{Z}_{b} u+\mathcal{R}_{b} u-V_{b} \psi+W_{b} \varphi=F_{0} \quad \text { in } \quad \Omega, \\
& \mathcal{Z}_{b}^{+} u+\mathcal{R}_{b}^{+} u-\mathcal{V}_{b} \psi+\mathcal{W}_{b} \varphi=F_{0}^{+}-\varphi_{0} \quad \text { on } \partial_{D} \Omega \text {, } \\
& T_{a}^{+} \mathcal{Z}_{b} u+T_{a}^{+} \mathcal{R}_{b} u-\mathcal{W}^{\prime}{ }_{a b} \psi+\mathcal{L}_{a b}^{+} \varphi=T_{a}^{+} F_{0}-\psi_{0} \quad \text { on } \quad \partial_{N} \Omega \text {. }
\end{aligned}
$$

Note that due to Lemma 1 , all terms of equation (4.35) belong to $H^{1,0}(\Omega ; \Delta)$ and their co-normal derivatives are well defined. System (4.35)-(4.37) can be rewritten in the form

$$
\mathcal{A}^{\mathcal{G} \mathcal{T}} \mathcal{U}=\mathcal{F}^{\mathcal{G} \mathcal{T}}
$$

where

$$
\begin{aligned}
\mathcal{U}^{\top}:= & {[u, \psi, \varphi] \in H^{1}(\Omega) \times \widetilde{H}^{-\frac{1}{2}}\left(\partial_{D} \Omega\right) \times \widetilde{H}^{\frac{1}{2}}\left(\partial_{N} \Omega\right), } \\
\mathcal{F}^{\mathcal{G} \mathcal{T}}:= & {\left[F_{0}, r_{\partial_{D} \Omega} F_{0}^{+}-\varphi_{0}, r_{\partial_{N} \Omega} T_{a}^{+} F_{0}-\psi_{0}\right]^{\top}, } \\
\mathcal{A}^{\mathcal{G T}}:= & {\left[\begin{array}{ccc}
I+\mathcal{Z}_{b}+\mathcal{R}_{b} & -V_{b} & W_{b} \\
r_{\partial_{D} \Omega}\left[\mathcal{Z}_{b}^{+}+\mathcal{R}_{b}^{+}\right] & -r_{\partial_{D} \Omega} \mathcal{V}_{b} & r_{\partial_{D} \Omega} \mathcal{W}_{b} \\
r_{\partial_{N} \Omega} T_{a}^{+}\left[\mathcal{Z}_{b}+\mathcal{R}_{b}\right] & -r_{\partial_{N} \Omega} \mathcal{W}_{a b}^{\prime} & r_{\partial_{N} \Omega} \mathcal{L}_{a b}^{+}
\end{array}\right] . }
\end{aligned}
$$




\subsubsection{Boundary-domain integral equation system $(\mathcal{G G})$}

To obtain another system, we will use equation (4.31) in $\Omega$ and equation (4.32), associated with the operator $\mathcal{G}$ on the whole boundary $\partial \Omega$, and arrive at the two-operator segregated BDIE system $(\mathcal{G G})$,

$$
\begin{aligned}
u+\mathcal{Z}_{b} u+\mathcal{R}_{b} u-V_{b} \psi+W_{b} \varphi=F_{0} & \text { in } \quad \Omega, \\
\frac{1}{2} \varphi+\mathcal{Z}_{b}^{+} u+\mathcal{R}_{b}^{+} u-\mathcal{V}_{b} \psi+\mathcal{W}_{b} \varphi=F_{0}^{+}-\Phi_{0} & \text { on } \quad \partial \Omega .
\end{aligned}
$$

System (4.38)-(4.39) can be written in the form

$$
\mathcal{A}^{\mathcal{G G}} \mathcal{U}=\mathcal{F}^{\mathcal{G G}},
$$

where

$$
\begin{aligned}
\mathcal{F}^{\mathcal{G G}} & :=\left[F_{0}, F_{0}^{+}-\Phi_{0}\right]^{\top}, \\
\mathcal{U}^{\top} & :=[u, \psi, \varphi] \in H^{1}(\Omega) \times \widetilde{H}^{-\frac{1}{2}}\left(\partial_{D} \Omega\right) \times \widetilde{H}^{\frac{1}{2}}\left(\partial_{N} \Omega\right), \\
\mathcal{A}^{\mathcal{G G}} & :=\left[\begin{array}{ccc}
I+\mathcal{Z}_{b}+\mathcal{R}_{b} & -V_{b} & W_{b} \\
\mathcal{Z}_{b}^{+}+\mathcal{R}_{b}^{+} & -\mathcal{V}_{b} & \frac{1}{2} I+\mathcal{W}_{b}
\end{array}\right] .
\end{aligned}
$$

\subsubsection{Boundary-domain integral equation system $(\mathcal{T} \mathcal{T})$}

To obtain one more system, we will use equation (4.31) in $\Omega$ and equation (4.33) on $\partial \Omega$ and arrive at the two-operator segregated BDIE system $(\mathcal{T} \mathcal{T})$,

$$
\begin{aligned}
& u+\mathcal{Z}_{b} u+\mathcal{R}_{b} u-V_{b} \psi+W_{b} \varphi=F_{0} \quad \text { in } \Omega, \\
& \left(1-\frac{a}{2 b}\right) \psi+T_{a}^{+} \mathcal{Z}_{b} u+T_{a}^{+} \mathcal{R}_{b} u-\mathcal{W}^{\prime}{ }_{a b} \psi+\mathcal{L}_{a b}^{+} \varphi= \\
& T_{a}^{+} F_{0}-\Psi_{0} \quad \text { on } \partial \Omega .
\end{aligned}
$$

System (4.40)-(4.41) can be written in the form

$$
\mathcal{A}^{\mathcal{T} \mathcal{T}} \mathcal{U}=\mathcal{F}^{\mathcal{T} \mathcal{T}},
$$

where

$$
\begin{aligned}
\mathcal{F}^{\mathcal{T} \mathcal{T}} & :=\left[F_{0}, T_{a}^{+} F_{0}^{+}-\Psi_{0}\right]^{\top}, \\
\mathcal{U}^{\top} & :=[u, \psi, \varphi] \in H^{1}(\Omega) \times \widetilde{H}^{-\frac{1}{2}}\left(\partial_{D} \Omega\right) \times \widetilde{H}^{\frac{1}{2}}\left(\partial_{N} \Omega\right), \\
\mathcal{A}^{\mathcal{T} \mathcal{T}} & :=\left[\begin{array}{ccc}
I+\mathcal{Z}_{b}+\mathcal{R}_{b} & -V_{b} & W_{b} \\
T_{a}^{+}\left[\mathcal{Z}_{b}+\mathcal{R}_{b}\right] & \left(1-\frac{a}{2 b}\right) I-\mathcal{W}_{a b}^{\prime} & \mathcal{L}_{a b}^{+}
\end{array}\right] .
\end{aligned}
$$




\subsubsection{Boundary-domain integral equation system $(\mathcal{T G})$}

To reduce BVP (4.5)-(4.7) to a BDIE system of "almost" the second kind (up to the spaces), we will use equation(4.31) in $\Omega$, the restriction of equation (4.33) on $\partial_{D} \Omega$, and the restriction of equation (4.32) on $\partial_{N} \Omega$. Then we arrive at the following two-operator segregated BDIE system $(\mathcal{T} \mathcal{G})$,

$$
\begin{aligned}
u+\mathcal{Z}_{b} u+\mathcal{R}_{b} u-V_{b} \psi+W_{b} \varphi=F_{0} & \text { in } \Omega \\
\left(1-\frac{a}{2 b}\right) T_{a}^{+} \mathcal{Z}_{b} u+T_{a}^{+} \mathcal{R}_{b} u-\mathcal{W}_{a b}^{\prime} \psi+\mathcal{L}_{a b}^{+} \varphi= & \\
T_{a}^{+} F_{0}-\Psi_{0} & \text { on } \partial_{D} \Omega \\
\frac{1}{2} \varphi+\mathcal{Z}_{b}^{+} u+\mathcal{R}_{b}^{+} u-\mathcal{V}_{a} \psi+\mathcal{W}_{a} \varphi=F_{0}^{+}-\Phi_{0} & \text { on } \partial_{N} \Omega .
\end{aligned}
$$

System (4.42)-(4.44) can be rewritten in the form

$$
\mathcal{A}^{\mathcal{T} \mathcal{G}}=\mathcal{F}^{\mathcal{T G}}
$$

where

$$
\begin{aligned}
& \mathcal{F}^{\mathcal{T} \mathcal{G}}:=\left[F_{0}, r_{\partial_{D} \Omega}\left(T_{a}^{+} F_{0}-\Psi_{0}\right), r_{\partial_{N} \Omega}\left(F_{0}^{+}-\Phi_{0}\right)\right]^{\top}, \\
& \mathcal{U}^{\top}:=[u, \psi, \varphi] \in H^{1}(\Omega) \times \widetilde{H}^{-\frac{1}{2}}\left(\partial_{D} \Omega\right) \times \widetilde{H}^{\frac{1}{2}}\left(\partial_{N} \Omega\right), \\
& \mathcal{A}^{\mathcal{T} \mathcal{G}}:=\left[\begin{array}{ccc}
I+\mathcal{Z}_{b}+\mathcal{R}_{b} & -V_{b} & W_{b} \\
r_{\partial_{D} \Omega} T_{a}^{+}\left[\mathcal{Z}_{b}+\mathcal{R}_{b}\right] & \left(1-\frac{a}{2 b}\right) I-r_{\partial_{D} \Omega} \mathcal{W}_{a b}^{\prime} & r_{\partial_{D} \Omega} \mathcal{L}_{a b}^{+} \\
r_{\partial_{N} \Omega}\left[\mathcal{Z}_{b}^{+}+\mathcal{R}_{b}^{+}\right] & -r_{\partial_{N} \Omega} \mathcal{V}_{b} & \frac{1}{2} I+r_{\partial_{N} \Omega} \mathcal{W}_{b}
\end{array}\right]
\end{aligned}
$$

\subsubsection{Equivalence and invertibility}

Using the arguments similar to the proofs of Theorems 5.2, 5.6, 5.9 and 5.12 in [CMN09], one can prove the following equivalence theorem.

Theorem 4. Let $f \in L_{2}(\Omega)$ and let $\Phi_{0} \in H^{\frac{1}{2}}(\partial \Omega)$ and $\Psi_{0} \in H^{-\frac{1}{2}}(\partial \Omega)$ be some fixed extensions of $\varphi_{0} \in H^{\frac{1}{2}}\left(\partial_{D} \Omega\right)$ and $\psi_{0} \in H^{-\frac{1}{2}}$, respectively.

(i) If some $u \in H^{1}(\Omega)$ solves the mixed $B V P$ (4.5)-(4.7) in $\Omega$, then the solution is unique and the triple $(u, \psi, \varphi) \in H^{1}(\Omega) \times \widetilde{H}^{-\frac{1}{2}}\left(\partial_{D} \Omega\right) \times \widetilde{H}^{\frac{1}{2}}\left(\partial_{N} \Omega\right)$, where

$$
\psi=T_{a}^{+} u-\Psi_{0}, \quad \varphi=u^{+}-\Phi_{0} \quad \text { on } \quad \partial \Omega
$$

solves BDIE systems $(\mathcal{G T}),(\mathcal{G G}),(\mathcal{T} \mathcal{T})$ and $(\mathcal{T} \mathcal{G})$.

(ii) Vise versa, if a triple $(u, \psi, \varphi) \in H^{1}(\Omega) \times \widetilde{H}^{-\frac{1}{2}}\left(\partial_{D} \Omega\right) \times \widetilde{H}^{\frac{1}{2}}\left(\partial_{N} \Omega\right)$ the solves BDIE system $(\mathcal{G} \mathcal{T})$ or $(\mathcal{G G})$ or $(\mathcal{T} \mathcal{T})$ or $(\mathcal{T} \mathcal{G})$, then the solution is unique, u solves BVP (4.5)-(4.7), and relations (4.45) hold. 
Application of the representation Lemma 5.13 and Corollary 5.14 as well as Corollary 5.16 about invertibility of the mixed BVP (4.5)-(4.7) operator, from [CMN09], along with the equivalence Theorem 4 above, lead to the following invertibility result.

Theorem 5. The following operators are continuously invertible,

$$
\begin{aligned}
& \mathcal{A}^{\mathcal{G G}}: H^{1,0}(\Omega ; \Delta) \times \widetilde{H}^{-\frac{1}{2}}\left(\partial_{D} \Omega\right) \times \widetilde{H}^{\frac{1}{2}}\left(\partial_{N} \Omega\right) \rightarrow H^{1,0}(\Omega ; \Delta) \times H^{\frac{1}{2}}(\partial \Omega), \\
& \mathcal{A}^{\mathcal{T} \mathcal{T}}: H^{1,0}(\Omega ; \Delta) \times \widetilde{H}^{-\frac{1}{2}}\left(\partial_{D} \Omega\right) \times \widetilde{H}^{\frac{1}{2}}\left(\partial_{N} \Omega\right) \rightarrow H^{1,0}(\Omega ; \Delta) \times H^{-\frac{1}{2}}(\partial \Omega), \\
& \mathcal{A}^{\mathcal{G T}}: H^{1,0}(\Omega ; \Delta) \times \widetilde{H}^{-\frac{1}{2}}\left(\partial_{D} \Omega\right) \times \widetilde{H}^{\frac{1}{2}}\left(\partial_{N} \Omega\right) \rightarrow \\
& H^{1,0}(\Omega ; \Delta) \times H^{\frac{1}{2}}\left(\partial_{D} \Omega\right) \times H^{-\frac{1}{2}}\left(\partial_{N} \Omega\right), \\
& \mathcal{A}^{\mathcal{T G}}: H^{1,0}(\Omega ; \Delta) \times \widetilde{H}^{-\frac{1}{2}}\left(\partial_{D} \Omega\right) \times \widetilde{H}^{\frac{1}{2}}\left(\partial_{N} \Omega\right) \rightarrow \\
& H^{1,0}(\Omega ; \Delta) \times H^{-\frac{1}{2}}\left(\partial_{D} \Omega\right) \times H^{\frac{1}{2}}\left(\partial_{N} \Omega\right) .
\end{aligned}
$$

\section{References}

[CMN09] O. Chakuda, S.E. Mikhailov and D. Natroshvili, Analysis of direct boundary-domain integral equations for a mixed BVP with variable coefficent, I: Equivalence and Invertibility. J. Int. Equat. Appl., 21, (2009).

[Co88] M. Costabel, Boundary integral operators on Lipschiz domains: Elementary results. SIAM journal on Mathematical Analysis, 19, 613-626 (1988).

[Gr85] P. Grisvard, Elliptic Problems in Nonsmooth Domains, Pitman, BostonLondon-Melbourne, (1985).

[LiMa72] J.L. Lions, E.Magenes, Non-Homogeneous Boundary Value Problems and Applications, Vol.1. Springer, Berlin - Heidberg - New York (1972).

[McL00] W. McLean, Strongly Elliptic Systems and Boundary Integral Equations. Cambrige University Press, Cambrige, (2000).

[Mi02] S.E. Mikhailov, Localized boundary-domain integral formulations for problems with variable coefficients, Int. J. Engineering Analysis with Boundary Elements, 26, 681-690 (2002).

[Mi05] S.E. Mikhailov, Localized direct boundary-domain integro-differential formulations for scalar nonlinear BVPs with variable coeffecients. Jornal of Engineering Mathematics, 51, 283-3002 (2005).

[Mi06] S.E. Mikhailov, Analysis of united boundary-domain integral and integrodifferential equations for a mixed BVP with variable coeffecients. Math. Meth. Appl. Sci. 29, 715-739 (2006).

[Mi07] S. E. Mikhailov, About traces, extensions and co-normal derivtive operators on Lipschitz domains. In: Constanda C. and Potapenko S. (eds), Integral Methods in Science and Engineering: Techniques and Applications, 149-160, Birkhäuser, Boston (2007).

[Mir70] C. Miranda, Partial Differential Equations of Elliptic Type., 2nd edition, Springer, Berlin-Heidelberg-New York (1970). 